\title{
Evaluation of the Genotoxicity of Aristolochic Acid in the Kidney and Liver of F344 gpt delta Transgenic Rat Using a 28-Day Repeated-dose Protocol: A Collaborative Study of the gpt delta Transgenic Rat Mutation Assay
}

\author{
Yuji Kawamura',6, Hiroyuki Hayashi², Osamu Tajima ${ }^{3}$, Sayuri Yamada ${ }^{3}$, \\ Tomomi Takayanagi ${ }^{4}$, Hisako Hori ${ }^{4}$, Wataru Fujii ${ }^{4}$, \\ Kenichi Masumura ${ }^{5}$ and Takehiko Nohmi ${ }^{5}$ \\ 1Toxicology Laboratory, Pharmaceutical Research Center, Meiji Seika Pharma Co., Ltd., Yokohama, Japan \\ ${ }^{2}$ Research Planning \& Management, R\&D Planning \& Management Dept., Meiji Seika Pharma Co., Ltd., Tokyo, Japan \\ ${ }^{3}$ Food Safety Assurance Center, Quality Assurance \& Environment Management Department, Kirin Group Office Co., \\ Ltd., Yokohama, Japan \\ ${ }^{4}$ Safety Science Institute, Quality Assurance Division, Suntory Business Expert Ltd., Osaka, Japan \\ ${ }^{5}$ Division of Genetics and Mutagenesis, National Institute of Health Sciences, Tokyo, Japan
}

(Received May 9, 2011; Revised June 20, 2011; Accepted July 5, 2011)

\begin{abstract}
Transgenic rat gene-mutation assays can be used to assess genotoxicity of chemicals in target organs for carcinogenicity. Since gene mutations in transgenes are genetically neutral and thus accumulate along with treatment periods, the assays are suitable for genotoxicity risk assessment of chemicals using repeated-dose treatment methodologies. However, few studies have been conducted to examine the suitability of the assays in repeat-dose treatment protocols. In order to prove the utility of the transgenic rat assays, we treated gpt delta rats with aristolochic acid at 0.3 and $1 \mathrm{mg} / \mathrm{kg}$ by gavage daily for 28 days, and autopsied the rats 3 days after the final treatment, which is a protocol recommended by the International Workshop on Genotoxicity Testing (IWGT). Aristolochic acid exists in herbs and some other plants, and is carcinogenic in the kidney, bladder and stomach in rats. The mutant frequency (MF) in both the kidney and the liver increased significantly in a dose-dependent manner when the rats were treated with aristolochic acid. We concluded that the gpt delta rat assay is sensitive enough to detect gene mutations induced by aristolochic acid and also that the 28-day repeated-dose protocol is suitable for assessing genotoxicity of chemicals.
\end{abstract}

Key words: F344 gpt delta transgenic rat, aristolochic acid, 28-day repeated-dose protocol, gpt assay

\section{Introduction}

Transgenic gene-mutation assays are of a high value for the assessment of in vivo genotoxicity $(1,2)$. In this method, mutations in reporter genes integrated in the rodent chromosomes can be identified in any organs/ tissues after the reporter genes are recovered from the rodent cells to bacterial cells. Transgenic gene-mutation assays are suitable for the risk assessment of potential genotoxic chemicals dosed via repeated-dose treatment, since mutations can be analyzed in various time points during treatment and sampling periods (3). In addition, mutations in the reporter genes accumulate over time as the treatments progress $(4,5)$. It is, therefore, expected that these assays enableus to assess the genotoxicity of chemicals with various dose levels, dosing periods and target organs.

Present issues to be solved for the use of transgenic gene-mutation assays include how the detection sensitivity can be confirmed and how the dosing periods can be standardized. In a genotoxicity assessment of 90 carcinogens, transgenic gene-mutation models are shown to have a high sensitivity and a good positive predictability (4). However, the majority of the 90 carcinogens assessed in that study are such strong mutagens that they could be used as positive controls in genotoxicity studies, and there are not enough data available on genotoxicants with a lower potency that allow assess-

${ }^{6}$ Correspondence to: Yuji Kawamura, Toxicology Laboratory, Pharmaceutical Research Center, Meiji Seika Pharma Co., Ltd., 760 Morooka-cho, Kohoku-ku, Yokohama 222-8567, Japan. Tel: +8145-545-3175, Fax: +81-45-545-3152, E-mail: yuuji.kawamura@ meiji.com 
ment of the method's sensitivity.

A recent trend regarding the use of experimental animals in toxicological studies focuses on replacement, reduction, and refinement (the ' $3 \mathrm{R}$ ' principles), and a movement towards these '3Rs' can be noted in presently reviewed guidelines for the assessment of genotoxicity. In addition to an in vivo bone marrow micronucleus test, we may select one more in vivo study instead of an in vitro study using cultured cells (6), the latter of which shows a comparatively high false-positive rate (7). It is now under discussion and, if conditions permit, we may integrate the in vivo genotoxicity assessment into a 28-day repeated-dose toxicity study for example. This approach would contribute to a reduction in the number of animals to be used experimentally. One of the promising candidates for the additional in vivo test is a test using a transgenic gene-mutation assay (8). However, nearly $70 \%$ of studies with transgenic genemutation assays have been conducted using a single dosing or repeated-dosing regimen within a 5-day period (3), and there are not enough data compiled for genotoxicity assessment using repeated treatment. This is contrast to the recommended protocol by the International Workshop on Genotoxicity Testing (IWGT), i.e., autopsy and sample collection on day 3 after the completion of a 28 -day repeated treatment $(28+3$ protocol $)$ $(9,10,11)$.

We initiated this study with the aim of testing the adequacy and detection capabilities of the IWGT-recommended general protocol for 28-day repeated-dose studies. For this work, we used F344 gpt delta rats, which were developed in Japan $(8,12)$. Aristolochic acid, which exists in herbs and some other plants (13), was used as the test substance, since it is genotoxic in vitro and in vivo $(14,15,16)$ and carcinogenic in rats $(17)$. In the carcinogenicity in rats, repeated treatment over 6-9 months induced tumors in the kidney, bladder, and stomach (17). In in vivo genotoxicity studies in Big Blue transgenic rats $(18,19)$, aristolochic acid was dosed orally for 12 weeks at the same doses used in the carcinogenicity study (17) and the frequency of $c I I$ mutation in the kidney $(18,19)$, a target organ for carcinogenicity, and the liver, a non-target organ, increased substantially.

In the current study, oral treatments with aristolochic acid increased gpt mutant frequency (MF) significantly in the kidney and the liver of F344 gpt delta rats in a dose-dependent manner, which suggests that four weeks treatment recommended by IWGT is sensitive enough to detect gene mutations.

\section{Materials and Methods}

F344 gpt delta rats: All animals were bred at Japan SLC, Inc. (Shizuoka, Japan). The F344 gpt delta transgenic rat strain was developed by backcrosses of the original SD gpt delta transgenic rat wtih wild-type
F344 rats. The gpt delta rat contains approximately 5 to 10 copies of the lambda EG10 transgene in chromosome 4 as a heterozygote (12). Male SD gpt delta rats were mated with wild-type F344 females to produce heterozygous F1 rats. F1 males (heterozygote for the transgene) were then backcrossed with F344 females. After 15 backcross matings, animals were designated as F344 gpt delta rats.

Chemical: Aristolochic acid (CAS\#313-67-7, purity $98 \%$, as 8 -methoxy-6-nitrophenanthro-(3,4-D)-1,3dioxolo-5-carboxylic acid, aristolochic acid-I) was purchased from Sigma-Aldrich (Tokyo, Japan). $N$-Ethyl$N$-nitrosourea (ENU, CAS\#759-73-9) was purchased from Nacalai Tesque, Inc. (Kyoto, Japan). The dosing solution of aristolochic acid was prepared by dissolving the chemical in purified water. The dosing solution of ENU was prepared by dissolving the chemical in saline.

Animals and treatments: The rats were used in the experiment at 7 weeks of age, after a 1-week acclimation period. The rats were housed individually in stainless steel cages, with free access to tap water and a CRF-1 pellet diet (Oriental Yeast Co., Ltd., Tokyo, Japan). The animal room conditions were maintained at a room temperature of $23 \pm 2{ }^{\circ} \mathrm{C}$, a relative humidity of $55 \pm$ $10 \%$, and a light-dark cycle of $12: 12 \mathrm{~h}$. The study protocol was approved by the Animal Care and Utilization Committee of Meiji Seika Pharma Co., Ltd. The treatments were conducted in accordance with the protocol recommended by the IWGT $(9,10,11)$. Five $g p t$ delta rats per group were dosed with aristolochic acid at 0 , 0.3 , or $1 \mathrm{mg} / \mathrm{kg}$ by gavage daily for 28 days, and necropsied 3 days after the final treatment for collection of the kidney and liver. The following parameters were monitored: clinical signs, body weight, food intake, hematology, blood chemistry, autopsy findings, organ weights, and histopathology. In addition, a positive control group was given an i.p. injection of $50 \mathrm{mg} / \mathrm{kg}$ ENU daily for 5 days, and autopsied 26 days after the final treatment for collection of the liver. The collected organs were immediately frozen in liquid nitrogen and stored at $-80^{\circ} \mathrm{C}$. The frozen samples were sent to Kirin Group Office Co., Ltd. (Lab. A) and Suntory Business Expert Ltd. (Lab. B) for gpt assays.

Detection of gpt mutation: The gpt assays were conducted in accordance with previously published methods in Lab. A and Lab. B separately $(1,20)$. Genomic DNA was extracted from the liver or the kidney using the RecoverEase ${ }^{\mathrm{TM}}$ DNA Isolation Kit (Agilent Technologies, Santa Clara, CA) and lambda EG10 phages were recovered with Transpack ${ }^{\circledR}$ Lambda Packaging Extract (Agilent Technologies). Escherichia coli YG6020 was infected with the phage, spread onto M9 salt plates containing chloramphenicol $(\mathrm{Cm})$ and 6-thioguanine (6-TG) (21), and then incubated for $72 \mathrm{~h}$ at $37^{\circ} \mathrm{C}$ for selection of the colonies harboring a plasmid 
carrying a chloramphenicol acetyltransferase gene and a mutated gpt gene. The mutant frequencies (MFs) of the gpt gene in the liver and kidney were calculated by dividing the number of confirmed 6-TG resistant colonies by the number of rescued plasmids.

Statistical analysis: The data for MFs were expressed as mean \pm SD. Statistically significant differences in MFs between the treated groups and the negative control were analyzed by Dunnett's multiple test or Steel's test. Statistically significant differences in MFs between the positive and negative control groups were analyzed by Welch's t-test. Differences in body weight, food intake, hematology, blood chemistry, and organ weights between the control and treated groups were analyzed by Dunnett's multiple test.

\section{Results}

gpt Mutations in the liver and kidney induced by aristolochic acid: In order to estimate the mutagenicity of aristolochic acid, gpt delta rats were treated orally for 28 days and mutations in the liver and kidney were analyzed in Lab. A and Lab. B (Fig. 1). Two laborato- a) Liver

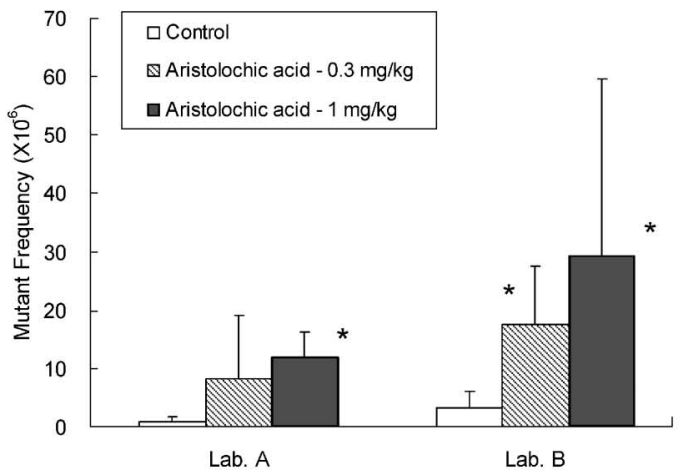

b) Kidney

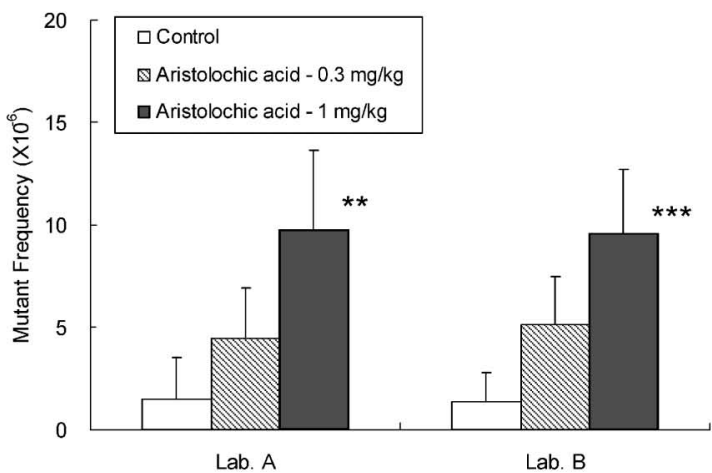

Fig. 1. Comparison between two laboratories in gpt mutant frequency of aristolochic acid-treated rats $(n=5)$ in a) Liver, b) Kidney. Values represent mean $+/-\mathrm{SD} .{ }^{*} p<0.05,{ }^{* *} p<0.01,{ }^{* * *} p<0.001$ (Steel test).

Table 1. $g p t$ Mutant frequencies in the liver of gpt delta rats treated with aristolochic acid

\begin{tabular}{|c|c|c|c|c|c|c|}
\hline \multirow{2}{*}{ Treatment } & \multirow{2}{*}{$\begin{array}{l}\text { Animal } \\
\text { No. }\end{array}$} & \multirow{2}{*}{$\begin{array}{c}\text { Total } \\
\text { population }\end{array}$} & \multirow{2}{*}{$\begin{array}{l}\text { Number of } \\
\text { mutants }\end{array}$} & \multicolumn{3}{|c|}{ Mutant frequency } \\
\hline & & & & $\left(\times 10^{-6}\right)$ & Average & SD \\
\hline \multirow{5}{*}{$\begin{array}{c}\text { Control } \\
\text { (Purified water) }\end{array}$} & 1 & $1,755,000$ & 6 & 3.42 & 1.92 & 1.02 \\
\hline & 2 & $1,158,000$ & 1 & 0.86 & & \\
\hline & 3 & $1,527,000$ & 2 & 1.31 & & \\
\hline & 4 & 654,000 & 1 & 1.53 & & \\
\hline & 5 & 813,000 & 2 & 2.46 & & \\
\hline \multirow{5}{*}{$\begin{array}{l}\text { Aristolochic acid } \\
\quad(0.3 \mathrm{mg} / \mathrm{kg})\end{array}$} & 11 & 606,000 & 5 & 8.25 & $12.28 * * *$ & 8.05 \\
\hline & 12 & 729,000 & 3 & 4.12 & & \\
\hline & 13 & 540,000 & 10 & 18.52 & & \\
\hline & 14 & 798,000 & 6 & 7.52 & & \\
\hline & 15 & 261,000 & 6 & 22.99 & & \\
\hline \multirow{5}{*}{$\begin{array}{l}\text { Aristolochic acid } \\
\quad(1 \mathrm{mg} / \mathrm{kg})\end{array}$} & 21 & $1,107,000$ & 28 & 25.29 & $15.29 * * *$ & 6.25 \\
\hline & 22 & $1,149,000$ & 14 & 12.18 & & \\
\hline & 23 & 888,000 & 15 & 16.89 & & \\
\hline & 24 & $1,104,000$ & 10 & 9.06 & & \\
\hline & 25 & $1,227,000$ & 16 & 13.04 & & \\
\hline \multirow{5}{*}{$\begin{array}{l}N \text {-Ethyl- } N \text {-nitrosourea } \\
(50 \mathrm{mg} / \mathrm{kg})\end{array}$} & 51 & 336,000 & 46 & 136.90 & $110.16^{\S \S}$ & 26.03 \\
\hline & 52 & 447,000 & 44 & 98.43 & & \\
\hline & 53 & 507,000 & 54 & 106.51 & & \\
\hline & 54 & 417,000 & 56 & 134.29 & & \\
\hline & 55 & 576,000 & 43 & 74.65 & & \\
\hline
\end{tabular}

${ }^{* *} p<0.01,{ }^{* * *} p<0.001$ (Dunnett test), ${ }^{\$ \S} p<0.001$ (welch's t-test). 
Table 2. $g p t$ Mutant frequencies in the kidney of gpt delta rats treated with aristolochic acid

\begin{tabular}{|c|c|c|c|c|c|c|}
\hline \multirow{2}{*}{ Treatment } & \multirow{2}{*}{$\begin{array}{c}\text { Animal } \\
\text { No. }\end{array}$} & \multirow{2}{*}{$\begin{array}{c}\text { Total } \\
\text { population }\end{array}$} & \multirow{2}{*}{$\begin{array}{l}\text { Number of } \\
\text { mutants }\end{array}$} & \multicolumn{3}{|c|}{ Mutant frequency } \\
\hline & & & & $\left(\times 10^{-6}\right)$ & Average & SD \\
\hline \multirow{5}{*}{$\begin{array}{c}\text { Control } \\
\text { (Purified water) }\end{array}$} & 1 & $1,020,000$ & 2 & 1.96 & \multirow[t]{5}{*}{1.69} & \multirow[t]{5}{*}{1.07} \\
\hline & 2 & 921,000 & 3 & 3.26 & & \\
\hline & 3 & $2,820,000$ & 1 & 0.35 & & \\
\hline & 4 & $1,656,000$ & 2 & 1.21 & & \\
\hline & 5 & 597,000 & 1 & 1.68 & & \\
\hline \multirow{5}{*}{$\begin{array}{l}\text { Aristolochic acid } \\
\quad(0.3 \mathrm{mg} / \mathrm{kg})\end{array}$} & 11 & $1,254,000$ & 6 & 4.78 & \multirow[t]{5}{*}{$4.82^{* *}$} & \multirow[t]{5}{*}{1.36} \\
\hline & 12 & 510,000 & 2 & 3.92 & & \\
\hline & 13 & 669,000 & 4 & 5.98 & & \\
\hline & 14 & $1,932,000$ & 6 & 3.11 & & \\
\hline & 15 & 474,000 & 3 & 6.33 & & \\
\hline \multirow{5}{*}{$\begin{array}{l}\text { Aristolochic acid } \\
\quad(1 \mathrm{mg} / \mathrm{kg})\end{array}$} & 21 & 954,000 & 10 & 10.48 & \multirow[t]{5}{*}{$9.14^{* * *}$} & \multirow[t]{5}{*}{3.60} \\
\hline & 22 & $1,965,000$ & 19 & 9.67 & & \\
\hline & 23 & $1,719,000$ & 9 & 5.24 & & \\
\hline & 24 & 987,000 & 14 & 14.18 & & \\
\hline & 25 & $1,797,000$ & 11 & 6.12 & & \\
\hline
\end{tabular}

${ }^{* *} p<0.01,{ }^{* * *} p<0.001$ (Dunnett test).

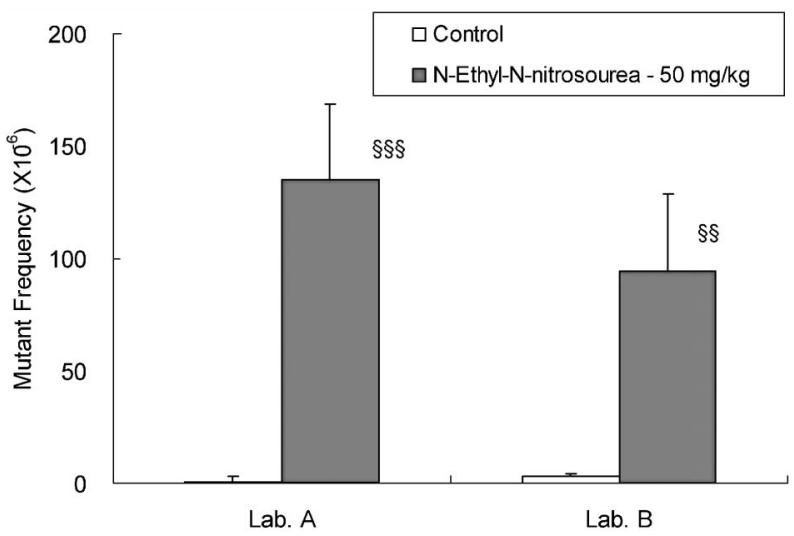

Fig. 2. Comparison between two laboratories in gpt mutant frequency of $N$-Ethyl- $N$-nitrosourea-treated rats $(n=5)$ in liver. Values represent mean $+/-\mathrm{SD} .{ }^{\S} p<0.01,{ }^{\S \S} p<0.001$ (welch's t-test).

ries generated quite similar results. In the liver, the mean numbers of gpt MFs in both Lab. A and Lab. B were $1.92 \pm 1.02,12.28 \pm 8.05$, and $15.29 \pm 6.25\left(\times 10^{6}\right)$ in the groups treated with $0,0.3$, and $1 \mathrm{mg} / \mathrm{kg}$ aristolochic acid, respectively (Table 1 ). The numbers of $g p t$ MFs in the liver in the aristolochic acid treatment groups increased in a dose-dependent manner to approximately $6.4-$ and 8.0 -fold that in the controls, for the 0.3 and $1 \mathrm{mg} / \mathrm{kg}$ treatments, respectively. These increases in MFs were statistically significant ( $p=0.00054$ and 0.00011 , respectively).

In the kidney, the mean numbers of gpt MFs in both Lab. A and Lab. B were $1.69 \pm 1.07,4.82 \pm 1.36$, and $9.14 \pm 3.60\left(\times 10^{6}\right)$ in the groups treated with $0,0.3$, and $1 \mathrm{mg} / \mathrm{kg}$ aristolochic acid, respectively (Table 2). The gpt MFs in the kidney in the aristolochic acid treatment groups increased in a dose-dependent manner to approximately 2.9 - and 5.4 -fold that in the controls. These increases in MFs were also statistically significant ( $p=$ 0.00843 and 0.00043 , respectively).

In the positive control group treated with $50 \mathrm{mg} / \mathrm{kg}$ ENU for 5 days, Lab. A and Lab. B showed very similar gpt MF in the liver of rats (Fig. 2). The gpt MF in the liver was $110.16 \pm 26.03\left(\times 10^{6}\right)$, which was approximately a 57.4-fold increase compared with the negative control group (Table 1). This increase in MFs was also statistically significant ( $p=0.00036)$.

Evaluation of the toxicity of aristolochic acid: A summary of the toxicity data generated for aristolochic acid is shown in Table 3. No mortalities occurred at any dose level during the dosing period. In the clinical observation, hematology, autopsy, and measurements of body weights, organ weights, and food intakes, no significant changes related to treatment with aristolochic acid were found at any dose. In the blood chemistry, the ALT value increased very slightly in the $1 \mathrm{mg} / \mathrm{kg}$ group. In the histopathology, very slight mononuclear infiltrations of the liver and very slight basophilic tubules in the kidney were observed in both of the $0.3 \mathrm{mg} / \mathrm{kg}$ and 1 $\mathrm{mg} / \mathrm{kg}$ groups.

\section{Discussion}

The aim of the present study was to assess the utility of gpt delta transgenic rats and the adequacy of the IWGT-recommended general protocol (9) through a genotoxicity risk assessment of aristolochic acid in the kidney and liver of rats. Aristolochic acid was administered orally to gpt delta rats at doses of 0.3 and 1 $\mathrm{mg} / \mathrm{kg}$ for 28 days, and the animals were autopsied 3 days after the last treatment so that the liver and kidney 
Table 3. Summary of toxicity data in gpt delta rats treated with aristolochic acid

\begin{tabular}{|c|c|c|c|}
\hline Animal species, age, sex & \multicolumn{3}{|c|}{ F344 gpt delta rat, 8 weeks old, male } \\
\hline Dosing method & \multicolumn{3}{|c|}{$\begin{array}{l}\text { Aristolochic acid was dissolved in water and administerd by oral gavage once a day for } 4 \text { weeks. } \\
\text { Dosing volume: } 10 \mathrm{~mL} / \mathrm{kg}\end{array}$} \\
\hline \multirow{2}{*}{ Dosing volume } & Vehicle control & \multicolumn{2}{|c|}{ Aristolochic acid (mg/kg/day) } \\
\hline & (Purified water) & 0.3 & 1 \\
\hline Number of animals & 5 & 5 & 5 \\
\hline Number of deaths & 0 & 0 & 0 \\
\hline Clinical signs & No abnormal signs & No abnormal signs & No abnormal signs \\
\hline Body weight & & - & - \\
\hline Food consumption & & - & - \\
\hline Hematology & & - & - \\
\hline Blood biochemistry & & - & Increase in ALT value* (1.4 fold) \\
\hline Autopsy & No remarkable changes & No remarkable changes & No remarkable changes \\
\hline Organ weight & & - & - \\
\hline Histonathology & No remarkable chanoes & $\begin{array}{l}\text { Mononuclear cell infiltration in the } \\
\text { liver ( } \pm: 2 / 5 \text { rats) }\end{array}$ & $\begin{array}{l}\text { Mononuclear cell infiltration in the } \\
\text { liver ( } \pm: 3 / 5 \text { rats) }\end{array}$ \\
\hline Histopatnology & No remarkable cnanges & $\begin{array}{l}\text { Basophilic change in the renal } \\
\text { tubules }( \pm: 4 / 5 \text { rats })\end{array}$ & $\begin{array}{l}\text { Basophilic change in the renal } \\
\text { tubules }( \pm: 3 / 5 \text { rats, }+: 1 / 5 \text { rat })\end{array}$ \\
\hline
\end{tabular}

-: No significant differneces compared with vehicle control. ${ }^{*} p<0.05$ (Dunnett test). Grade in histopathology: $\pm:$ Very slight, $+:$ Slight.

could be collected for the detection of mutations with the gpt assay. A significant and dose-dependent increase in the MF was noted in the kidney, a carcinogenicity target organ, in the groups treated with aristolochic acid compared with the MF in the negative control group ( $\mathrm{T}$ able 2). In a previous genotoxicity study using Big Blue transgenic rats $(18,19)$, aristolochic acid was administered repeatedly for 12 weeks at 0.1 and $1 \mathrm{mg} / \mathrm{kg}$ and the frequency of $c I I$ mutations in the kidney increased approximately 3 - and 8 -fold compared with the control group, respectively. The increases in gpt MF in the present study were approximately 3 - and 5 -fold, at the dose levels of 0.3 and $1 \mathrm{mg} / \mathrm{kg}$, respectively, indicating an almost equivalent ability to detect mutations as in the assessment with Big Blue rats treated for 12 weeks, and also demonstrating that a 28 -day dosing period is sufficient for detection.

The rat carcinogenicity study was conducted at dose levels of $0.1,1.0$, and $10 \mathrm{mg} / \mathrm{kg}(17)$. That study revealed adenoma in the kidney, and hyperplasia and carcinoma in the renal pelvis after 6 months of treatment at $10 \mathrm{mg} / \mathrm{kg}$, adenoma in the kidney and hyperplasia in the renal pelvis after 9 months of treatment at $1 \mathrm{mg} / \mathrm{kg}$, and hyperplasia in the renal pelvis after 12 months of treatment at $0.1 \mathrm{mg} / \mathrm{kg}$. An increased MF in the kidney was observed in gpt delta rats treated at $1 \mathrm{mg} / \mathrm{kg}$ (Table 2), which strongly suggested that the carcinogenicity observed in the kidney was related to the genotoxicity. However, gpt MF was also increased in the liver, a non- target organ of carcinogenicity (Table 1). Similar results are reported with Big Blue rat (19). Accordingly, aristolochic acid was judged to have genotoxicity in the liver. In the rat carcinogenicity study with aristolochic acid, the maximum duration of administration was 9, 9 and 16 months in the groups treated with $0.1,1$, and 10 $\mathrm{mg} / \mathrm{kg}$ aristolochic acid, respectively (17). That study duration of 9-16 months was rather short compared with the 2 year duration that is typical of carcinogenicity studies. This might be a reason why no induction of liver cancer was detected. Alternatively, other factors such as accelerating cell proliferation may be required for the induction of cancer in the liver. Further work is needed to discuss the relationship between genotoxicity and carcinogenicity in the liver of rats treated with aristolochic acid.

In the present study, we also carried out hematology, blood chemistry, autopsy, and organ weight measurements to examine the general toxicity of aristolochic acid using the same animals. These analyses did not identify any abnormalities except a slight increase in ALT that might be effects of aristolochic acid administration. The histopathology revealed basophilic changes in the kidney and mononuclear infiltration in the liver. However, these effects were very slight, and thus we judged that aristolochic acid did not induce significant tissue damage in the study. In a 28-day repeated-dose toxicity study using gpt delta rats, it would be possible to assess not only genotoxicity but also general toxicity. 
This is another merit of the IWGT-recommended protocol and gpt delta rats. However, in ordinary repeateddose toxicity studies, autopsy is conducted one day after the final treatment $(28+1$ protocol). In transgenic genemutation assays, in contrast, sampling time of about 3 days after the final treatment is set as a period in which DNA adducts are converted to mutations. The difference of the sampling time might be a roadblock to integrate transgenic rat assays into 28 -day repeat dose toxicity assays. However, if we sample the organs one day after the final treatment $(28+1$ protocol $)$, it will be almost equivalent to 26 days administration time plus 3 days sampling time $(26+3$ protocol). Because the administration periods of 26 days and 28 days are not substantially different in terms of total dose, we expect that the results from sampling one day after the last treatment $(28+1$ protocol) will be very similar to those from the assays conducted with the protocol recommended by IWGT $(28+3$ protocol). In addition, if we set the dosing period for general toxicity studies to be 1 month $(30+1$ protocol) instead of 4 weeks $(28+3$ protocol), we would expect to have similar results to those generated if sampling was conducted 3 days after the 28 day of treatment. On the other hand, the length of the recovery period after the final treatment is very important in general toxicity evaluation, because the result may be different depending on the length. Since the basophilic changes in the kidney, which were noted 3 days after the final treatment with aristolochic acid, were regenerative, acute tissue injury might be observed in the case where necropsy was conducted 1 day after the final treatment. Therefore, we suggest that $28+3$ protocol recommended by IWGT should not be rigid and also that the protocols can be flexibly adapted to repeat dose toxicity protocols such as $28+1$ or $30+1$.

For studies using transgenic rat gene-mutation assays, we need to further promote the standardization of experimental procedures. There have not been any reports comparing the results for the same chemical evaluated at different laboratories. In the present study, the gpt assay on organs originating from the same animal was conducted in two different laboratories and the results were combined for assessment (Figs. 1 and 2). Figure 1 shows the results of the gpt assay of aristolochic acid in each laboratory. The results of the analyses on the mutations in the kidney in the two laboratories were similar to each other and almost comparable. As for the liver, some differences were noted in the statistical analyses in the low dose groups, but otherwise we noted similar tendencies. Figure 2 shows the gpt assay results of the positive control (ENU) in each laboratory. The results of the analyses on mutations in the positive control in the two laboratories were highly comparable. In transgenic gene-mutation assays, the recovery of the reporter genes and the method for identifaication of mutated-colonies are influential factors on the results of the study. It is, therefore, expected that international validation of standardized technical procedures among laboratories will proceed in the future.

In conclusion, the genotoxicity of aristolochic acid was sensitively detected in the kidney and the liver in the 28-day repeated treatment study using gpt delta rats, and thus the adequacy of the IWGT-recommended protocol $(28+3)$ was confirmed.

Acknowledgments: We thank organizers of the collaborative study group for the transgenic rat mutation assay for reviewing the manuscript and giving us helpful suggestions. In addition, we thank Meiji Seika Pharma's members who helped us in a conduct of animal experiments or a review of the manuscript.

\section{References}

1 Nohmi T, Suzuki T, Masumura K. Recent advances in the protocols of transgenic mouse mutation assays. Mutat Res. 2000; 455: 191-215.

2 Nohmi T, Masumura K. Molecular nature of intrachromosomal deletions and base substitutions induced by environmental mutagens. Environ Mol Mutagen. 2005; 45: 150-61.

3 Lambert IB, Singer TM, Boucher SE, Douglas GR. Detailed review of transgenic rodent mutation assays. Mutat Res. 2005; 590: 1-280.

4 Suzuki T, Hayashi M, Ochiai M, Wakabayashi K, Ushijima T, Sugimura T, Nagao M, Sofuni T. Organ variation in the mutagenicity of MeIQ in Big Blue lacI transgenic mice. Mutat Res. 1996; 369: 45-9.

5 de Vries A, van Oostrom CT, Dortant PM, Beems RB, van Kreijl CF, Capel PJ, van Steeg H. Spontaneous liver tumors and benzo[a]pyrene-induced lymphomas in XPAdeficient mice. Mol Carcinog. 1997; 19: 46-53.

6 Hayashi M. Update on the maintenance of the ICH S2 genetic toxicology. Pharm Regul Sci. 2008; 39: 515-21.

7 Kirkland D, Aardema M, Henderson L, Müller L. Evaluation of the ability of a battery of three in vitro genotoxicity tests to discriminate rodent carcinogens and non-carcinogens I. Sensitivity, specificity and relative predictivity. Mutat Res. 2005; 584(1-2): 1-256.

8 Toyoda-Hokaiwado N, Inoue T, Masumura K, Hayashi H, Kawamura Y, Kurata Y, Takamune M, Yamada M, Sanada H, Umemura T, Nishikawa A, Nohmi T. Integration of in vivo genotoxicity and short-term carcinogenicity assays using F344 gpt delta transgenic rats: in vivo mutagenicity of 2,4-diaminotoluene and 2,6-diaminotoluene structural isomers. Toxicol Sci. 2010; 114: 71-8.

9 Thybaud V, Dean S, Nohmi T, de Boer J, Douglas GR, Glickman BW, Gorelick NJ, Heddle JA, Heflich RH, Lambert I, Martus HJ, Mirsalis JC, Suzuki T, Yajima N. In vivo transgenic mutation assays. Mutat Res. 2003; 540: 141-51.

10 Heddle JA, Dean S, Nohmi T, Boerrigter M, Casciano D, Douglas GR, Glickman BW, Gorelick NJ, Mirsalis JC, Martus HJ, Skopek TR, Thybaud V, Tindall KR, Yajima 
$\mathrm{N}$. In vivo transgenic mutation assays. Environ Mol Mutagen. 2000; 35: 253-9.

11 International Program on Chemical Safety (2006): Transgenic animal mutagenicity assays. Environmental Health Criteria 233, World Health Organization (WHO), Geneva.

12 Hayashi H, Kondo H, Masumura K, Shindo Y, Nohmi T. Novel transgenic rat for in vivo genotoxicity assays using 6-thioguanine and Spi- selection. Environ Mol Mutagen. 2003; 41: 253-9.

13 Kite GC, Yule MA, Leon C, Simmonds MS. Detecting aristolochic acids in herbal remedies by liquid chromatography/serial mass spectrometry. Rapid Commun Mass Spectrom. 2002; 16: 585-90.

14 Arlt VM, Stiborova M, Schmeiser HH. Aristolochic acid as a probable human cancer hazard in herbal remedies: a review. Mutagenesis. 2002; 17: 265-77.

15 Schmeiser HH, Stiborova M, Arlt VM. Chemical and molecular basis of the carcinogenicity of Aristolochia plants. Curr Opin Drug Discov Devel. 2009; 12: 141-8.

16 Zhang H, Cifone MA, Murli H, Erexson GL, Mecchi
MS, Lawlor TE. Application of simplified in vitro screening tests to detect genotoxicity of aristolochic acid. Food Chem Toxicol. 2004; 42: 2021-8.

17 Mengs U, Lang Wm Poch J-A, The carcinogenic action of aristolochic acid in rats. Arch Toxicol 1982; 51: 107-19.

18 Chen L, Mei N, Yao L, Chen T. Mutations induced by carcinogenic doses of aristolochic acid in kidney of Big Blue transgenic rats. Toxicol Lett. 2006; 165: 250-6.

19 Mei N, Arlt VM, Phillips DH, Heflich RH, Chen T. DNA adduct formation and mutation induction by aristolochic acid in rat kidney and liver. Mutat Res. 2006; 602: 83-91.

20 Nohmi T, Katoh M, Suzuki H, Matsui M, Yamada M, Watanabe M, Suzuki M, Horiya N, Ueda O, Shibuya T, Ikeda $\mathrm{H}$, Sofuni T. A new transgenic mouse mutagenesis test system using Spi- and 6-thioguanine selections. Environ Mol Mutagen. 1996; 28: 465-70.

21 Seidman MM, Dixon K, Razzaque A, Zagursky RJ, Berman ML. A shuttle vector plasmid for studying carcinogen-induced point mutations in mammalian cells. Gene. 1985; 38: 233-7. 